\title{
Feasibility of a Novel ECG Electrode Placement Method in Newborn Infants
}

\author{
Caroline Henry $^{\mathrm{a}}$ Lara Shipley $^{\mathrm{a}}$ Stephen Morgan $^{\mathrm{b}}$ John A. Crowe ${ }^{\mathrm{b}}$ \\ James Carpenter ${ }^{c}$ Barrie Hayes-Gill ${ }^{b}$ Don Sharkey ${ }^{a}$ \\ ${ }^{a}$ Centre for Perinatal Research, School of Medicine, University of Nottingham, Nottingham, UK; ${ }^{b}$ Faculty of \\ Engineering, University of Nottingham, Nottingham, UK; 'SurePulse Medical Ltd., Nottingham, UK
}

\section{Keywords}

Neonatology $\cdot$ Resuscitation $\cdot$ Heart rate .

Electrocardiograms · Technology

\begin{abstract}
Background: International newborn resuscitation guidelines recommend electrocardiogram (ECG) heart rate (HR) monitoring at birth. We evaluated the application time of pre-set ECG electrodes fixed to a polyethene patch allowing adhesive-free attachment to the wet skin of the newborn chest. Objectives: Using a three-electrode pre-set ECG patch configuration, application success was calculated using video analysis and measured at three time points, the time to (1) apply electrodes; (2) detect recognizable QRS complexes after application; and (3) display a HR after application. Method: A prospective observational study in two UK tertiary maternity units was undertaken with 71 newborns including 23 who required resuscitation. Results: The median (IQR) time for ECG patch application was 8 (6-10) seconds, detection of recognizable QRS complexes 8 (2-12) seconds, and time to output HR was 23 (15-37) seconds. Conclusion: Pre-set ECG chest electrodes allow rapid $\mathrm{HR}$ information at birth without electrode detachment or compromising skin integrity.
\end{abstract}

(c) 2022 The Author(s).

Published by S. Karger AG, Basel

karger@karger.com

www.karger.com/neo

Karger $\stackrel{\text { ' }}{=}$

BOPEN ACCESS
C) 2022 The Author(s).

Published by S. Karger AG, Basel

This is an Open Access article licensed under the Creative Common (http://www.karger.com/Services/OpenAccessLicense), applicable to the online version of the article only. Usage and distribution for commercial purposes requires written permission. Attribution-NonCommercial-4.0 International License (CC BY-NC)

\section{Introduction}

Accurate heart rate (HR) assessment is a crucial component of newborn resuscitation algorithms. Electrocardiograms (ECG) provide rapid continuous monitoring of HR during resuscitation [1], and international guidelines suggest their use in the delivery room $[1,2]$. Ideally, ECG must be quickly sited by healthcare professionals (HCPs) and subsequent electrode detachment avoided. Conventional ECG electrodes require individual placement following skin drying and can take $26 \mathrm{~s}$ to site [3]. Polyethene bags are used for thermal care in newborn preterm infants, adhering to the skin through surface tension but allowing removal from delicate skin without causing shearing damage. These benefits could save HCP time and aid stabilization with more reliable monitoring. We aimed to evaluate the application time of pre-set ECG electrodes integrated onto a polyethene chest patch for newborn infants.

\section{Materials and Methods}

This is a sub-analysis of a prospective observational study group from a larger delivery room trial, HeartLight [4].

\section{Study Population}

The study was conducted in two tertiary maternity centres at Nottingham University Hospitals. Infants $>22$ weeks gestation

Correspondence to:

Don Sharkey, don.sharkey@ nottingham.ac.uk 
Table 1. Characteristics of newborns included

\begin{tabular}{lllll}
\hline Gestational group & $<32$ weeks $(n=9)$ & $32-36$ weeks $(n=38)$ & $\geq 37$ weeks $(n=20)$ & All infants $(n=67)$ \\
\hline Gestation, weeks & $28^{+5}\left(25^{+0}-31^{+2}\right)$ & $35^{+1}\left(32^{+2}-36^{+0}\right)$ & $39^{+0}\left(37^{+3}-42^{+0}\right)$ & $35^{+5}\left(25^{+0}-42^{+0}\right)$ \\
Male, $n(\%)$ & $6(66)$ & $15(40)$ & $7(35)$ & $28(42)$ \\
Birthweight, kg & $1.16(0.80-1.69)$ & $2.25(1.01-4.10)$ & $3.38(2.04-4.47)$ & $2.44(0.8-4.47)$ \\
Caesarean section, $n(\%)$ & $8(88)$ & $29(76)$ & $20(100)$ & $57(85)$ \\
Resuscitation needed, $n(\%)$ & $7(78)$ & $14(37)$ & $2(10)$ & $23(34)$ \\
\hline
\end{tabular}

Data are median (range) unless stated otherwise. Resuscitation refers to the need for at least positive pressure mask ventilation.

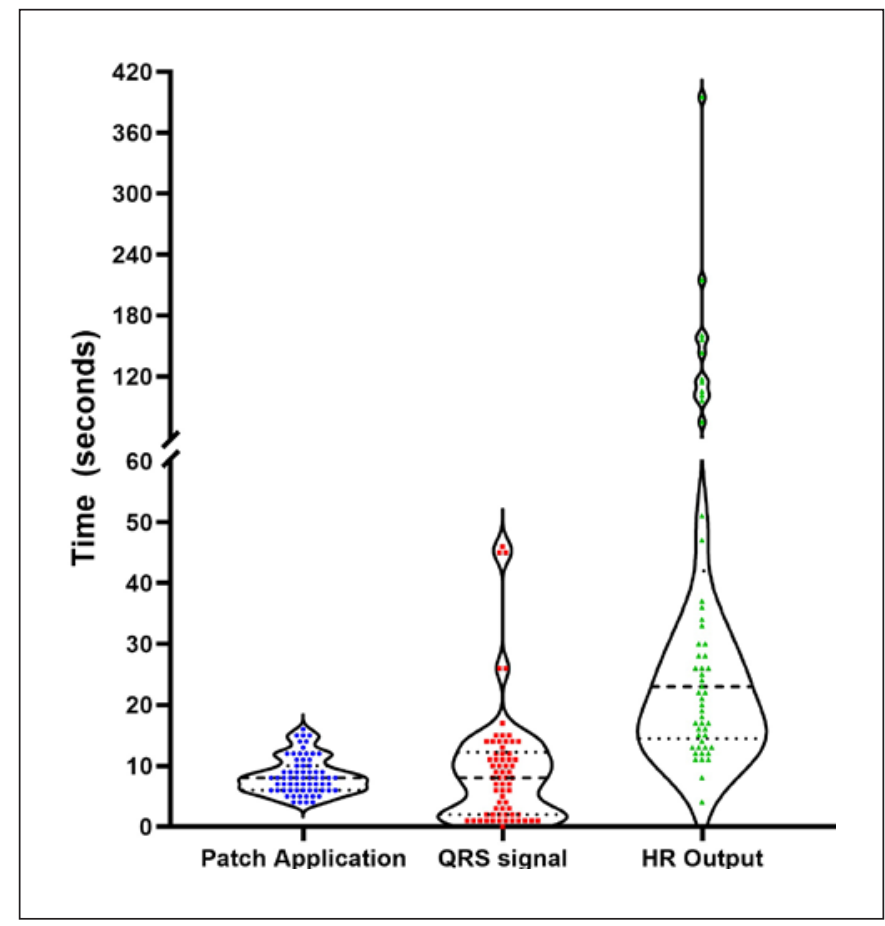

Fig. 1. Violin plot of time taken to apply the ECG patch, obtain a QRS signal and output a HR on the Carescape B450 monitor in all 67 infants. Dashed line is the median value and dotted lines are the IQR.

were eligible for inclusion if the clinical team were offering active management at birth. Sample size was determined by the aims of the HeartLight protocol.

\section{Study Design}

Prior to birth, ECG electrodes (SKINTACT; Leonhard Lang, Innsbruck, Austria) were attached to a $7 \mathrm{~cm}$ by $7 \mathrm{~cm}$ square patch of low-density polyethene wrap normally used for preterm thermoregulation (online suppl. material 1; for all online suppl. material, see www.karger.com/doi/10.1159/000521530). Three holes (diameter $1 \mathrm{~cm}$ ) were made in the patch to allow electrode contact with the skin via ECG conduction gel placed onto the electrodes to avoid adhesive attachment to the skin. The electrodes were connected to a Carescape Monitor B450 (General Electric Healthcare, Chicago, IL, USA) outputting HR data every $5 \mathrm{~s}$.

A Novel ECG Placement Method
Following birth, the infant was brought to the resuscitaire. Infants $\geq 32$ weeks were dried except the chest area for patch placement, infants $<32$ weeks were placed wet into a plastic bag. Their heads were dried and a cap placed (HeartLight trial). The ECG patch was then applied on the chest (online suppl. Video 1) and a pulse oximeter (Carescape B450) attached on the right hand/wrist. A webcam recorded the study along with LabVIEW 2014 (National Instruments, Austin, TX, USA) which collected real-time data.

\section{Outcome Measures}

Three time points were calculated: (1) time to apply the patch once picked up; (2) time to detect recognizable QRS complexes after placement (online suppl. material 2); and (3) time after placement until the ECG HR value output. If a piece of equipment (for example, a stethoscope on the chest) delayed placing the pre-set electrodes, this time was subtracted from the deployment time. Success rate, a measure of device reliability, is defined as the percentage of time during which the patch, once attached to the infant, reports a HR. Data are presented as median (range and/or IQR).

\section{Results}

\section{Population}

Seventy-one newborns were recruited to the study, four were excluded: video obscured (one), HeartLight protocol deviation (two), loss of HR output data (one). This left 67 newborns included in the analysis (Table 1).

\section{Outcomes}

The median times for ECG application was $8 \mathrm{~s}$ (IQR 6-10 s) (Fig. 1) and a recognizable QRS to be displayed after application was $8 \mathrm{~s}$ (IQR 2-12 s), with all infants displaying a QRS signal by $46 \mathrm{~s}$. The median time for a HR value output from the monitor was $23 \mathrm{~s}$ (IQR 15-37 s). Within 1 min of arrival on the resuscitaire, all infants had a recognizable QRS signal and $80 \%$ a HR value. There were no electrode detachments and HR output success rate was $95 \%$ ( $n=8,440$ of 8,907 timepoints).

Fourteen $(21 \%)$ newborns were $<1,500 \mathrm{~g}$ had an application time of $11 \mathrm{~s}$ (IQR 7-12 s) and time to QRS signal 
of $4 \mathrm{~s}$ (IQR $1-7 \mathrm{~s}$ ). Infants $\geq 1,500 \mathrm{~g}$ had an application time of $8 \mathrm{~s}$ (IQR 6-9 s) and time to QRS signal of $9 \mathrm{~s}$ (IQR 3-14 s). There were no issues removing the patch or any areas of skin damage.

\section{Discussion}

Utilizing the natural surface tension properties of wet newborn skin, we found a simple, biocompatible lowdensity polyethene patch allowed quick and easy electrode attachment across a range of infants at birth without any detachments, providing rapid HR information. International guidance suggests the use of continuous ECG monitoring. Despite the many advantages ECG continuous monitoring provides, the attachment of each electrode can be time consuming, often requiring a dedicated individual to apply them, and they can easily detach especially with wet or vernix covered skin.

Application time of ECG electrodes at birth has previously been studied with ranges between $17.1 \mathrm{~s}$ and $114 \mathrm{~s}$ [2, $5,6]$. This wide range may be accounted for by differing start times, for example, time of birth rather than being placed on the resuscitaire or handling the electrodes, and the number of HCPs involved in the resuscitation. Application time in this study was $8 \mathrm{~s}$ with a narrow range following placement by a single HCP. Previously, a similar approach placed a patch on the back using adhesive tape [7]. Following initial evaluation of back placement, we opted to use the chest to allow repositioning if needed and avoid any damage to fragile skin from adhesive. If incorrectly placed or with poor signal quality, back placement would be more difficult to readjust without disrupting resuscitation efforts. Our success rate was a HR output for $95 \%$ of the timepoints during the study without any electrode displacements demonstrating the excellent attachment technique.

Following application, the initial QRS signal was obtained in a median of $8 \mathrm{~s}$ and by $46 \mathrm{~s}$ for all 67 infants. There was more variability and delay with the displayed HR which is dependent on the type of monitor and algorithms used to analyse the signal. As such we did not have access to the raw data and so were unable to interrogate delays further. Katheria et al. [3] reported in two studies obtaining a visible or audible ECG signal within $2 \mathrm{~s}$ (1-4 s) of application and within $66 \mathrm{~s}( \pm 20 \mathrm{~s})$ from being placed on the resuscitation bed [8], although in the later study the ECG signal quality could only be used for 40 of the 86 infants. Iglesias et al. [6] reported an ECG HR at $26 \mathrm{~s}$ after application, and Gulati et al. [7] reported HR values at 29 s but with a $30 \%$ failure rate at $1 \mathrm{~min}$ from time of arrival onto the resuscitation bed. Our HR output failure rate was $20 \%$ at $1 \mathrm{~min}$, but all infants had a QRS signal by 46 s. Median time to QRS signal was $8 \mathrm{~s}$ and as such, it may be more helpful to HCPs to be able to see the ECG trace on the monitor rather than just a displayed number obtained by the algorithm.

Previous delays in ECG HR display have been attributed to not drying the skin well enough and thick vernix preventing ECG sensor attachment [9]. Iglesias et al. [6] reported no data in the first $30 \mathrm{~s}$ after birth, and Gulati et al. [7] found that infants vigorous at birth took longer to pick up a signal because of movement artefacts. In our study, it was quicker to deploy the patch in larger infants, possibly because they were not placed in a polyethene bag, but the time to QRS and HR acquisition was slightly longer than smaller infants. The amount of conductive gel may have varied which could contribute to differences in data acquisition. The placement of the leads was not modified for different infant sizes as we wanted to see if a single product could be used no matter what the size of the baby.

Limitations of the present study were the low number of vaginal births and no infants required chest compressions. It is not possible to establish if there are any potential differences between caesarean or vaginal deliveries as a larger number of infants would be required. It is unlikely the patch would interfere with chest compressions as plastic wraps are used for most preterm infants without reported problems although there could be signal interference with or without the patch. Ideally, comparison with standard electrode placement would be undertaken for comparison, but this was not the focus of the main study and should form part of a future study.

\section{Conclusion}

Using a pre-set ECG electrode chest patch enables quick, continuous monitoring and avoids single electrode detachment or potential skin stripping injuries from adhesives. The patch allows rapid HR signal acquisition and reliable output reporting with no electrode detachements. This approach warrants further development and evaluation to understand if these benefits result in improved monitoring and stabilization outcomes.

\section{Acknowledgments}

Carole Ward and Siavash Mirahmadi helped with data acquisition and analysis. 


\section{Statement of Ethics}

This study was approved by NHS Health Research Authority 15/YH/0522, HeartLight study Clinicaltrials.gov NCT02701920, $3 / 3 / 2016$. Written informed parental consent was obtained for the publication of the details of the medical cases and any accompanying images or videos prior to infant enrollment.

\section{Conflict of Interest Statement}

S.M. and J.Cr. are shareholders, J.Ca. is a shareholder, and C.E.O., B.H.G., and D.S. are shareholders and nonexecutive directors of SurePulse Medical Ltd., who are developing newborn vital sign devices.

\section{Funding Sources}

This study was supported by Innovate UK: MRC-MC_ PC_15012. The funder had no role in preparing data or manuscript.

\section{Author Contributions}

C.H.: designed protocol, acquired, analysed and interpreted data, and drafted manuscript. L.S.: protocol design, acquired data. S.M., J.Cr., J.Ca., and B.H.G.: protocol development and data analysis. D.S.: designed protocol and interpreted data. All the authors revised and approved the final manuscript.

\section{Data Availability Statement}

All data generated or analyzed during this study are available upon reasonable request by contacting the corresponding author.

\section{References}

1 Aziz K, Lee HC, Escobedo MB, Hoover AV, Kamath-Rayne BD, Kapadia VS, et al. Part 5: neonatal resuscitation: 2020 American Heart Association guidelines for cardiopulmonary resuscitation and emergency cardiovascular care. Circulation. 2020;142(16 Suppl 2):S524-50.

2 Madar J, Roehr CC, Ainsworth S, Ersdal H, Morley C, Rüdiger M, et al. European Resuscitation Council guidelines 2021: newborn resuscitation and support of transition of infants at birth. Resuscitation. 2021;161:291-326.

3 Katheria A, Rich W, Finer N. Electrocardiogram provides a continuous heart rate faster than oximetry during neonatal resuscitation. Pediatrics. 2012;130(5):e1177-81.
4 Henry C, Shipley L, Ward C, Mirahmadi S, Liu C, Morgan S, et al. Accurate neonatal heart rate monitoring using a new wireless, cap mounted device. Acta Paediatr. 2021; 110(1):72-8.

5 Kamlin CO, O’Donnell CP, Everest NJ, Davis PG, Morley CJ. Accuracy of clinical assessment of infant heart rate in the delivery room. Resuscitation. 2006;71(3):319-21.

6 Iglesias B, Rodriguez MJ, Aleo E, Criado E, Herranz G, Moro M, et al. Pulse oximetry versus electrocardiogram for heart rate assessment during resuscitation of the preterm infant. An Pediatr. 2016;84(5):271-7.
7 Gulati R, Zayek M, Eyal F. Presetting ECG electrodes for earlier heart rate detection in the delivery room. Resuscitation. 2018;128: 83-7.

8 Katheria A, Arnell K, Brown M, Hassen K, Maldonado M, Rich W, et al. A pilot randomized controlled trial of EKG for neonatal resuscitation. PLoS One. 2017;12(11): e0187730.

9 Mizumoto H, Tomotaki S, Shibata H, Ueda K, Akashi R, Uchio H, et al. Electrocardiogram shows reliable heart rates much earlier than pulse oximetry during neonatal resuscitation. Pediatr Int. 2012;54(2):205-7. 